\title{
Resolution Analysis of Grey Scale Image using Spherical Harmonics (SPH) Method
}

\author{
Muhammad Abbas Khan and Piao Yan* \\ School of Electronic and Information Engineering, Changchun University of Science and Technology, \\ Changchun-130022, China; engineerabbaskhan111@gmail.com, pioyan66@126.com
}

\begin{abstract}
Objectives/Aim: To used spherical harmonics coefficients and spherical texture mapping techniques to increases or decreases the reconstructed image resolution from unit sphere. Methods: We used mat lab as a simulation tool to mapped the grey scale image which is a strings of zeros and ones and also called monochrome or black in white image to mapped a grey scale image to a unit sphere by spherical texture mapping. The spherical Texture mapping used a Spherical co-ordinate system for mapping of grey scale image to extract picture information to unit sphere, we also used mat lab for reconstruction of an image from unit sphere using spherical harmonics coefficients. Findings: We analyzed that by increasing the spherical harmonics coefficients the PSNR value increases and the MSE value decreases because more number of pixels of grey scale image mapped to unit sphere and the image resolution of grey scale improved, while decreasing the value of Spherical harmonics coefficient the PSNR value decreases and MSE value increases, so less numbers of pixels are mapped to unit sphere and the grey scale image resolution decreases. In past it can be used for computer graphics, magnetic field of star and planetary bodies, geoids and for gravitational fields. In this paper we used it for resolution analysis of grey scale image from a unit sphere. Application/Improvements: In future it can also be used for medical images to increase or decrease his resolution, and for color images which consist of three elementary colors red, green, and blue.
\end{abstract}

Keywords: Euclidean Distance (ED), Grey Scale Image, Mean Square Error (MSE), Peak Signal To Noise Ratio (PSNR), Spherical Harmonics (SH), Spherical Texture Mapping, Unit Sphere

\section{Introduction}

The Spherical Harmonics (SPH) is a function, which is defined mostly on the surface of sphere. It is mostly used to solve Partial Differential Equation's (PDE). The spherical harmonics are the orthogonal functions on the surface of sphere, Spherical harmonics obeys Laplace's Equation, and the functions which satisfy Laplace Equation called Harmonic and hence got the name spherical harmonics. In this paper we employ Spherical Texture Mapping, Which Texture the gray scale image to Spherical co-ordinates on the unit sphere and then employ Spherical harmonics method to reconstruct the image by different values of spherical harmonics coefficients " $C_{l^{m}}$ ".

\section{Unit Sphere}

The unit Sphere mean's that it has radius of one i.e. $r=1$ and centered at the origin" o" as shown in Figure 1. Mathematically

$\mathrm{x}^{2}+\mathrm{y}^{2}=1$

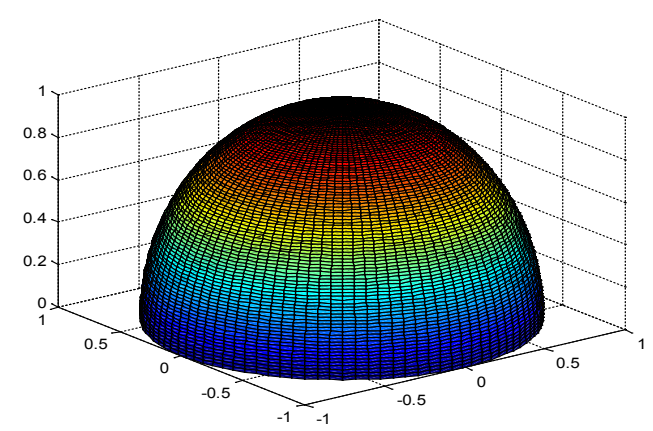

Figure 1. Unit sphere.

\section{Grey Scale Image}

The grey scale image is also called a black in white image, 
which consist of two colors string's Black and white. The black color strings are represented by strings of zero's (0's), while the White color are represented by strings of one's (1's) as shown in Figure 2. The grey scale image carries intensity information consists of shades of grey.

$$
\left[\begin{array}{l}
100 \\
101 \\
110
\end{array}\right]
$$

Figure 2. Grey scale image matrix.

\section{Spherical Texture Mapping}

In Spherical Texture mapping, we mapped the grey scale image which is the Stings of zero's and one's into the unit sphere of radius one $r=1$. As Mathematically mapping is f: A B. So the grey scale image co-ordinates are changed to spherical co-ordinates on the unit sphere. The spherical co-ordinates is a co-ordinate system for three dimensional space, which determines the position of point by three numbers $(r, \Theta, \Phi)$. Where " $r$ " is the radius " $\Theta$ " is the polar angle and " $\Phi$ " is the azimuthal angle Figure 3 ,

Mathematically

$\mathrm{r}=\sqrt{x^{2}+y^{2}+z^{2}}$

$\Theta=\arccos z / r$

$\Phi=\arctan y / x$

\section{PSNR}

PSNR is the Parameter used to find the image quality. It is a measure of peak error. The higher values of PSNR means that the image quality of reconstructed image is better and if the PSNR value is less it means that the image quality or reconstructed image is not good. PSNR is measured in decibel $(\mathrm{dB})$.

Mathematically

PSNR $=10 \log _{10}\left(\mathrm{maxi}^{2} /\right.$ Mean square error $)$

Where in equation (5) the" maxif "is the maximum number of pixel's in grey scale image. When the image is of 8 bits then the maxi ${ }^{2}$ is equal to 255 .

$\operatorname{maxi}^{2}=2^{b}-1$. Where " $b$ " is the numbers of bits per sample. In this paper we increases or decreases the PSNR value by increasing or decreasing the coefficient " $C_{l^{m}}$ " value of spherical harmonic. which will increase or decrease the number's of pixel's to unit sphere and the reconstructed image quality will increase or decrease $\$ y$ varying the spherical harmonic coefficient values " $C_{l^{\prime}}$,

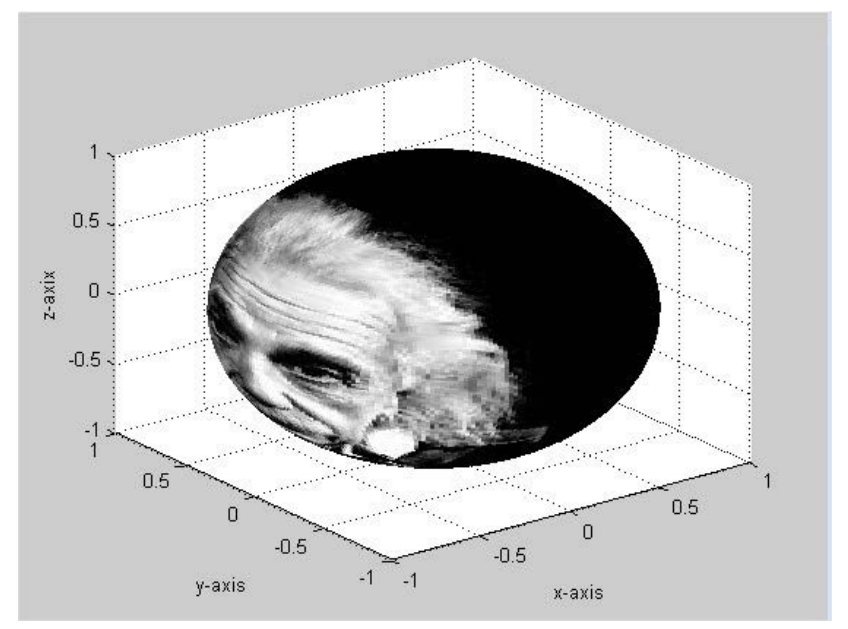

Figure 3. Spherical harmonics texture mapping.

\section{MSE}

MSE is a another parameter to find image quality, which shows the cumulative error between original grey scale image and the reconstruction image, higher the value of MSE lower will be the PSNR and poor will be the image quality. While lower the value of MSE higher will be the PSNR and the image resolution or quality will improved. In this paper the MSE value will increase if the spherical harmonics coefficients " $C_{l^{m}}$ " have minimum value, because less number of pixels are mapped to a unit sphere and PSNR will decrease and the image quality is not good. But when the spherical harmonics coefficient " $C_{l^{m}}$ " have maximum values maximum numbers of pixels are mapped to a unit sphere, while in this case the Mean square error is minimum and PSNR is maximum and the image quality will improved, when we increase the spherical harmonics coefficients " $C_{l^{m}}$ " to maximum values.

Mathematically

$M S E=\frac{1}{m n \sum_{i=0}^{m-1} I(i, j)}-\sum_{j=0}^{n-1} h(i, j)$

Where in equation (6) the $\mathrm{m}, \mathrm{n}$ are row's and column's of $m^{\star} n$ grey scale image, while $i, j$ shows the position of pixel's in grey scale image. The "I" shows the original image and " $h$ " is the reconstructed image, 


\section{Euclidean Distance (ED)}

The Euclidean Distance (ED) shows the distance between pixels. If the Euclidean Distance (ED) between pixels is high, PSNR will be minimum and MSE will be high and the image quality will be not too good, but if Euclidean Distance (ED) between the pixels is less the PSNR will be maximum and MSE will be minimum and the picture quality improved. In this paper we increases or decreases the spherical harmonics coefficient's " $C_{l^{m}}$ " to increase or decrease the Euclidean Distance (ED) between pixels of grey scale image. As we increase spherical harmonics coefficient's " $C_{l^{m}}$ " the Euclidean Distance (ED) between pixels of grey scale image will be minimum on a unit sphere and the PSNR will be high and MSE will be minimum and the picture quality will improve, while decreases spherical harmonics coefficient's " $C_{l^{m}}$ "the Euclidean Distance (ED) between pixels of grey scale image will be maximum on a unit sphere and PSNR will be low and MSE will be maximum and the image quality will be not too good.

Mathematically

$(E d) d(h, j)=\sqrt{\left(h_{1}-j_{1}\right)^{2}+\left(h_{2}-j_{2}\right)^{2}+\ldots+\left(h_{n}-j_{n}\right)^{n}}$

where " $h$ " and " $j$ " in equation (7) are the position's of pixels on unit sphere. It's unit is meter denoted by “' $\mathrm{m}$ '.

\section{Spherical Harmonics (SPH)}

It is the orthogonal basis function on the surface of unit sphere, orthogonal means that they also pull in every direction. The Spherical Harmonics (SPH) is a function, which is defined mostly on the surface of sphere. The spherical harmonics are the orthogonal functions on the sphere, which is mostly used to define functions defined on the surface of sphere. Spherical harmonics obeys Laplace's Equation, which is the second ordered partial differential equation, the main solution of Laplace equation is harmonic functions, and the functions which satisfy Laplace Equation called Harmonic and hence got the name spherical harmonics,

Mathematically

Laplace equation

$$
\nabla^{2} \emptyset=0
$$

Where in equation (8) the " $\nabla$ " is the Laplace operator and " $\emptyset$ is the scalar function. The Laplace's equations are mostly called the steady state heat equation. Spherical harmonics,

Mathematically

$$
\mathrm{Y}_{1}^{\mathrm{m}}(\Theta, \Phi)=\mathrm{N}_{1}^{\mathrm{m}} \mathrm{P}_{1}^{\mathrm{m}} \cos \Theta \mathrm{e}^{\mathrm{im} \Phi}
$$

Where in equation (9) " $\mathrm{Y}_{1}^{\mathrm{m}}(\Theta, \Phi)$ " is the basis function with polar angle " $\Theta$ " from $0<\theta<\pi$ and $\Phi$ is the azimuthal angle from $0<\Phi<2 \pi$. The " $\mathrm{N}_{1}^{\mathrm{m}}$ " is

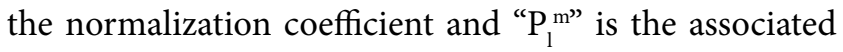
Legendre Polynomial's," $\cos \Theta \mathrm{e}^{\mathrm{im} \Phi}$ are cosine component and Euler's formula. The associated Legendre Polynomials

Mathematically

$\mathrm{P}_{1}^{\mathrm{m}}(\mathrm{x})=(-1)^{\mathrm{m}} \sqrt{\left(1-x^{2}\right)^{\mathrm{m}} / 2 ! ! !}(\mathrm{d} / \mathrm{dx})^{1+\mathrm{m}}\left(\mathrm{x}^{2}-1\right)^{1}$

where in equation(10) have range" 1 " $0 \leq l \leq N$ $\leq l \leq N$ and $0 \leq m \leq l$. Any function on the surface of unit sphere can be represented mathematically in terms of spherical harmonics

$$
\mathrm{f}(\Theta, \Phi)=\sum_{l=0}^{\infty} \sum_{l=0}^{m=l} l, m
$$

where in equation (11) " $C_{l^{m}}$ " is the spherical harmonic

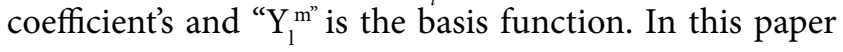
we use the spherical harmonic coefficient " $C_{l^{m}}$ " for reconstruction of image from unit sphere to check image resolution by PSNR, MSE values of the grey scale image. Spherical harmonic coefficient " $C_{l^{\prime}}$ ”

$$
\begin{gathered}
\text { Mathematically } \\
c_{l^{m}}=\int_{0}^{2 \pi} \int_{0}^{\pi} \mathrm{f}(\Theta, \Phi) \mathrm{Y}_{1}^{\mathrm{m}}(\Theta, \Phi) \sin \Theta \mathrm{d} \Theta \mathrm{d} \Phi
\end{gathered}
$$

where in equation (12) the " $f(\Theta, \Phi)$ " is the function on the surface of unit sphere in terms of spherical harmonics

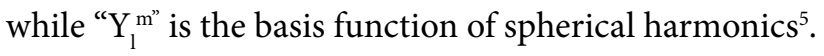

\section{Matlab Simulation Results}

Original grey scale image: The original image as shown in figure is a grey scale image, which is also called monochrome image as shown in Figure 4. 


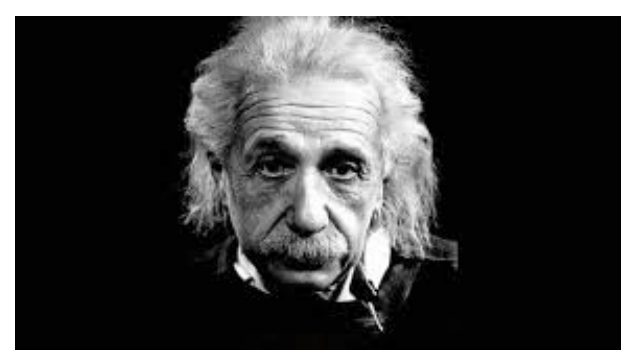

Figure 4. Original grey scale image.

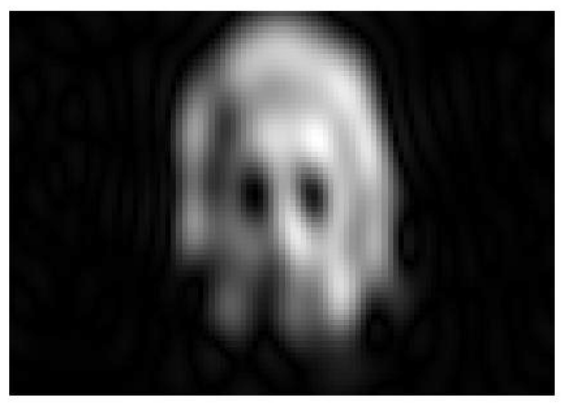

Figure 4(a). $\quad C_{l^{m}}=15$.

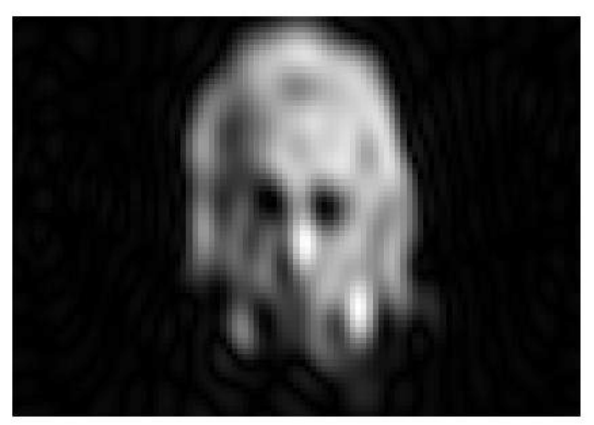

Figure $4(\mathrm{~b}) . \quad C_{l^{m}}=20$.

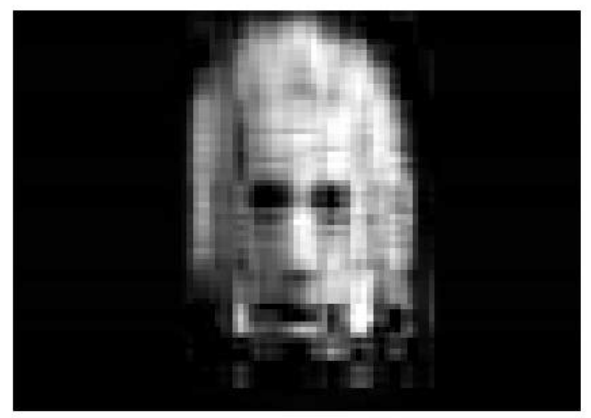

Figure 4(c). $\quad C_{l^{m}}=25$.

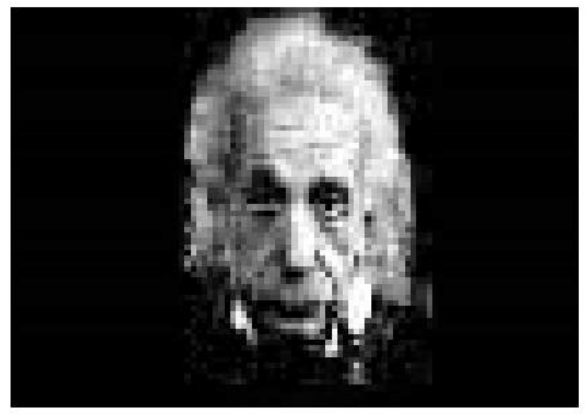

Figure $4(\mathrm{~d}) . \quad C_{l^{m}}=30$.

In this paper we use matlab as a simulation tool. So we analyzed that as, we in increase's the spherical harmonic coefficients " $C_{l^{m}}$ " the PSNR value increase's and the MSE value decrease's and the picture quality improved. So as we in decrease's the spherical harmonic coefficient's $C_{l^{m}}$ "the PSNR value decrease's and the MSE value increase's and the picture quality is not good as show in Figure 4. In the first two cases in Figure 4 (a), (b) the $C_{l^{m}}$ value is less so the picture quality and, the PSNR value is not too good, while the MSE value is high, because in this two cases the numbers of pixels of grey scale image are less mapped to the unit sphere. While in figure 4 (c), (d) the $C_{l^{m}}$ value is high so the picture quality and the PSNR value is too good, while the MSE value is less, because in this two cases the numbers of pixels of grey scale image are mapped more to unit sphere. So we concluded as we increases or decrease the spherical harmonicpqoefficient's $C_{l^{m}}$ the picture quality increases or decreases ${ }^{\circ}$

Figure 5 are the real life picture which mapped to sphere and apply spherical harmonics coefficient's of $C_{l^{m}=40}$ so the picture quality improved, and look like original grey scale image.

As shown in Table 1 comparisons of PSNR, MSE, Spherical harmonics coefficients. $C_{l^{m}}$

Table 1. Comparison of PSNR,MSE, Spherical harmonics coefficients and image quality

\begin{tabular}{lrrrl}
\hline S. NO. & $C_{l^{m}}$ & (PSNR) dB & (MSE) & Image quality \\
\hline 1 & 15 & 33.2550 & 4.7260 & Very bad \\
2 & 20 & 33.9417 & 4.0349 & Not good \\
3 & 25 & 34.3244 & 3.6945 & Medium \\
4 & 30 & 34.3959 & 3.6342 & Good \\
\hline
\end{tabular}




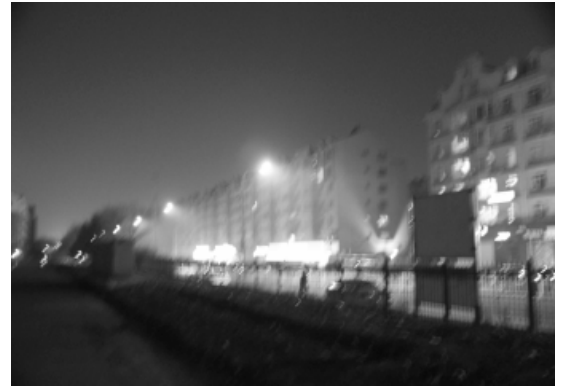

Figure 5(a).

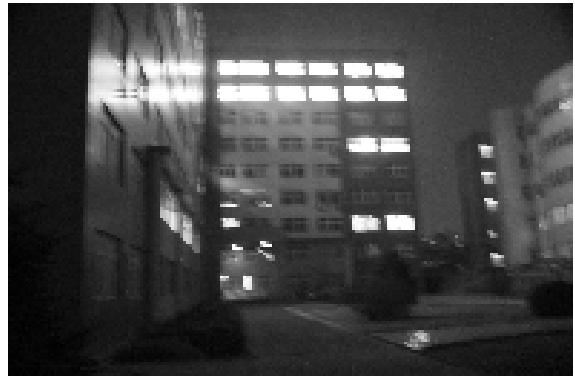

Figure 5(b). Original grey scale image’s.

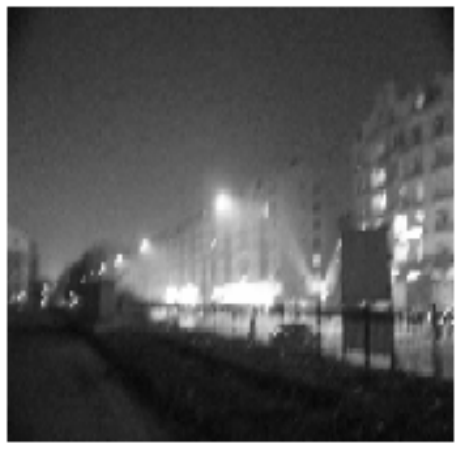

Figure 5(c). Reconstructed image $C_{l^{m}}=40$.

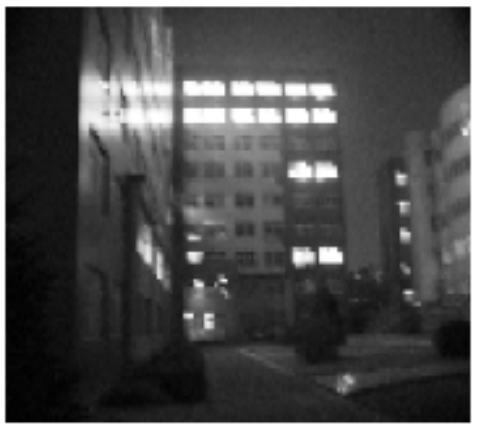

Figure 5(d). Reconstructed image $C_{l^{m}}=40$.

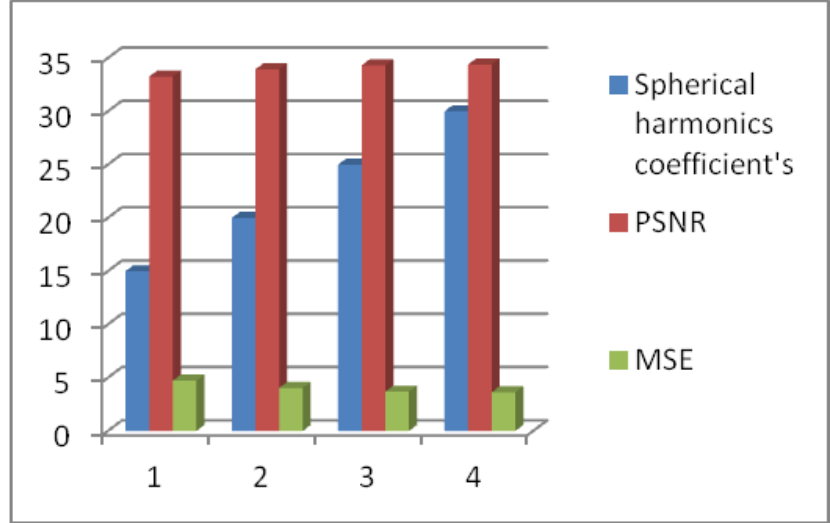

Graph 1. Comparison of PSNR,MSE, Spherical harmonics coefficients .

\section{Conclusion Remarks}

In this paper we use a Spherical harmonic method, in which we increased or decreased the spherical harmonic Coefficients " $C_{l}$ ". So we concluded that by increasing the Spherical Harmonics coefficients " $C_{l^{m}}$ " the PSNR value increases, while the MSE value decreases, and the picture quality will improve, because in this case more numbers of pixels are mapped to the unit sphere. While decreases the Spherical harmonics coefficients " $C_{l^{m}}$ " the PSNR value decreases and the MSE value increase's and the picture quality are not good, while in this case the numbers of pixels are less mapped to the unit sphere.

\section{References}

1. Frederick MW, Venkat D. Texture mapping 3D model's of real-world Scenes. ACM Computing Surveys. 1997 Dec; 29(4):325-65.

2. Pooja K, Yuvraj S. Comparison of different image enhancement techniques based upon PSNR and MSE. International Journal of applied Engineering and Research. 2002; 7(2):111.

3. Kaiwen Z, Shuozhong W, Xinpen Z. A new metric for quality assessment of digital images based on weighted-mean square error. Proceedings of SPIE; 2002 Jul. p. 1-6.

4. Jing L, Bao LL. An adaptive image Euclidean distance. Elsevier. 2008 Jul; 42:349-57.

5. Wojceich J, Natan AC, Henrick WJ. Importance sampling spherical harmonics. Computer Graphics Forum. 2009 Apr; 28(2):577-86.

6. Bennet MA, Nair RR, Mahalakshmi V, Janakiraman V. Performance and analysis of ground-glass pattern detection in 
lung disease based on high-resolution computed tomography. Indian Journal of Science and Technology. 2016 Jan; 9(2):1-7.

7. Sakthivel M, Dhayalan D, Babu MS, Murugan JS. Robust aircraft recognition using high resolution reconstructed images. Indian Journal of Science and Technology. 2016 Jan; 9(3):1-7.
8. Ashima, Mohana R. Anaphora resolution in Hindi: Issues and directions. Indian Journal of Science and Technology. 2016 Aug; 9(32):1-8.

9. Jung YH, Park K, Chae JM, Jung SY. Resolution of ellipse in coordinate noun phrases. Indian Journal of Science and Technology. 2015; 8(21):1-5. 